\title{
Comparación de las propiedades antioxidantes y contenido de po- lifenoles de extractos acuosos de las algas marinas Bryothamnion triquetrum y Halimeda opuntia
}

\section{Comparation of antioxidants properties and polyphenols content of aqueous extract from seaweeds Bryothamnion triquetrum and Halimeda opuntia}

Daylín Díaz Gutierrez ${ }^{1}$ - Wendy Méndez Ortega ${ }^{1}$ - Ana Mara de Oliveira e Silva ${ }^{2}$ Claudina Zaldívar Muñoz ${ }^{1}$ Jorge Mancini-Filho ${ }^{3}$ Alexis Vidal Novoa ${ }^{1}$

1. Grupo de Farmacología y Toxicología, Facultad de Biología, Universidad de La Habana, La Habana, Cuba.

2. Area de Nutrición, Universidad Federal de Sergipe, Aracaju, Sergipe, Brasil.

3. Departamento de Alimentos y Nutrición Experimental, Facultad de Ciencias Farmacéuticas, Universidad de São Paulo, São Paulo, Brasil.

\section{Artículo de Revisión \\ Review Article}

\section{Correspondencia} Correspondence

Dr. Alexis Vidal Novoa

Grupo de Farmacología y Toxicología,

Departamento de Bioquímica

Facultad de Biología, Universidad

de La Habana

Calle 25 No. 455 e/ J e I Vedado,

CP 14000

La Habana, Cuba

alexis.vidal@infomed.sld.cu

alexisvidal@fbio.uh.cu

Financiación

Fundings

Este trabajo fue financiado parcialmente por CNPq (Brasil) a través del Proyecto No. 401852/2012-1

\section{Conflicto de interés}

Competing interest

En esta investigación no existen conflictos de intereses de tipo económicos y/o de otra índole.

Received: 01.02.2015 Accepted: 28.03.2015

\section{creative}

commons

LICENSE 3.0 UNPORTED.

\section{RESUMEN}

Objetivos. Evaluar y comparar las propiedades antioxidantes mediante ensayos in vitro de extractos acuosos de las algas roja Bryothamnion triquetrum y verde Halimeda opuntia y su relación con el contenido de polifenoles.

Material y Métodos. Se utilizaron las técnicas in vitro: DPPH, Capacidad reductora, Inhibición de la peroxidación lipídica e inhibición de la hemólisis inducida por AAPH.

Resultados. B. triquetrum: DPPH; $\mathrm{CI}_{50}=1,15 \pm 0,06$, capacidad reductora a concentración; $128 \mathrm{mg} / \mathrm{mL}$, $\mathrm{DO}=2,798$, inhibición de la peroxidación lipídica; $\mathrm{CI}_{50}=5,09 \pm 0,25$ e inhibición de la hemólisis con $12 \mathrm{mg} /$ $\mathrm{mL} ; 35 \%$. H. opuntia: $\mathrm{DPPH} ; \mathrm{CI}_{50}=12,34 \pm 0,30 \mathrm{mg} / \mathrm{mL}$, capacidad reductora; $\mathrm{DO}=0,800$, inhibición de la peroxidación lipídica; $\mathrm{CI}_{50}=1,25 \pm 0,31 \mathrm{mg} / \mathrm{mL}$ e inhibición de la hemólisis; $82 \%$.

Conclusiones. Los resultados evidencian que $B$ triquetrum resulto mucho más eficiente en los ensayos de DPPH y Capacidad reductora mientras H opuntia resulta más eficiente en Inhibición de la peroxidación lipídica e inhibición de la hemólisis. Se discuten algunos aspectos acerca de sus posibles mecanismos de acción.

Palabras claves: algas marinas, antioxidantes Halimeda opuntia, Bryothamnion triquetrum, polifenoles.

\section{ABSTRACT}

Objective. To evaluate and compare the antioxidant activity displayed by seaweed $\mathrm{H}$. opuntia and $B$. triquetrum using different experimental in vitro antioxidant assessment models.

Material and Methods. The following techniques are utilized: DPPH, Reducing power, inhibition of lipid peroxidation and inhibition of haemolysis.

Results. B. triquetrum: DPPH; $\mathrm{IC}_{50}=1.15 \pm 0.06$, Reducing power (concentration $128 \mathrm{mg} / \mathrm{mL}$ ), $\mathrm{OD}=2.798$, inhibition of lipid peroxidation; $\mathrm{IC}_{50}=5.09 \pm 0.25$ and inhibition of haemolysis; with $12 \mathrm{mg} / \mathrm{mL} ; 35 \%$. $H$. opuntia: $\mathrm{DPPH} ; \mathrm{IC}_{50}=12.34 \pm 0.30 \mathrm{mg} / \mathrm{mL}$, reducing power; $\mathrm{OD}=0.800$, inhibition of lipid peroxidation; $\mathrm{IC}_{50}=1.25 \pm 0.31 \mathrm{mg} / \mathrm{mL}$, inhibition of lipid peroxidation; $\mathrm{IC}_{50}=1.25 \pm 0.31 \mathrm{mg} / \mathrm{mL}$ and inhibition of haemolysis; $82 \%$.

Conclusion. It was demonstrated that B.triquetrum extract was more effective than $H$. opuntia in reducing power and DPPH assays while H.opuntia was more effective in inhibition of lipid peroxidation in rat brain homogenates and the inhibition of red blood cell (RBC) haemolysis induced by AAPH. We discuss some aspects about their possible mechanisms of action.

Keywords: seaweed, antioxidants, Halimeda opuntia, Bryothamnion triquetrum, polyphenol. 


\section{INTRODUCCIÓN}

Las algas marinas forman parte de la dieta tradicional en algunas regiones del mundo, sobre todo asiática, con un alto valor nutricional como fuentes de proteínas, minerales, vitaminas y fibras dietéticas ${ }^{1}$ y desde épocas ancestrales han sido utilizadas como fitofármacos contra diferentes patologías ${ }^{2}$. En los últimos años, las investigaciones acerca de posibles propiedades terapéuticas de las algas marinas han cobrado una marcada importancia, motivado en cierta medida por su contenido de metabolitos secundarios bioactivos $^{3}$. Diferentes estudios in vitro y en modelos animales así como investigaciones epidemiológicas, han evidenciado una relación directa e inversa entre el consumo de algas y la incidencia de algunas patologías ${ }^{4}$.

La síntesis de determinados metabolitos secundarios en las algas marinas puede ser explicada como un mecanismo de defensa contra circunstancias adversas del medio ambiente entre los que se pueden citar la temperatura, luz solar, $\mathrm{pH}$, estrés oxidativo y presencia de peces herbívoros. En los mares tropicales las algas están expuestas a una alta incidencia de luz solar lo que puede conducir a la formación de radicales libres, de manera que la ausencia de daños oxidativos de sus componentes estructurales y fisiológicos evidencian que estos organismos presentan un eficiente sistema de defensas antioxidantes ${ }^{5}$.

Las propiedades antioxidantes de las algas marinas pueden ser explicadas por la presencia de moléculas como carotenoides, aminoácidos tipo micosporinas, terpenoides y polisacáridos sulfatados, aunque la mayoría de los investigadores consideran a los compuestos polifenólicos como los ácidos fenólicos y cinámicos, florotaninos y bromofenoles, entre los principales responsables de esta propiedad ${ }^{6}$.

En trabajos previos de nuestro Laboratorio, con especies de los géneros Bryothamnion y Halimeda se demostraron propiedades antioxidantes en sistemas libres de células, en modelos experimentales in vitro y en modelos animales ${ }^{7-11}$.

Considerando estos antecedentes, el objetivo de este trabajo fue evaluar y comparar las propiedades antioxidantes mediante ensayos in vitro de extractos acuosos de las algas marinas roja Bryothamnion triquetrum y verde Halimeda opuntia y su relación con el contenido de polifenoles así como esclarecer sus posibles mecanismos de acción.

\section{MATERIAL Y MÉTODOS}

Colecta y preparación de los extractos acuosos de las algas Bryothamnion triquetrum y Halimeda opuntia

Especimenes de las algas marinas roja Bryothamnion triquetrum (S.G.Gmelin) Howe y verde, Halimeda opuntia (Lin- naeus) Lamouroux fueron colectadas en el área del Instituto de Oceanología (La Habana, Cuba) en febrero/2014. Los especimenes fueron identificados y autenticados en el Laboratorio de Algas del Centro de Investigaciones Marinas de la Universidad de La Habana, donde se depositó una muestra.

Las algas se secaron durante 7-10 días a temperatura ambiente. Para la preparación de los extractos las algas secas fueron molidas en mortero y tamizadas. Posteriormente se procedió a obtener el extracto acuoso del mismo realizando una extracción con agua destilada 1:5 (p/v) durante 5 h con agitación constante a temperatura ambiente. Seguidamente se centrifugó a $800 \mathrm{~g}$ en una centrífuga HealForce (Beckman GS-GKR, USA) a $4^{\circ} \mathrm{C}$ durante 20 minutos. El sobrenadante se liofilizó y posteriormente fue conservado a $-20^{\circ} \mathrm{C}$ hasta su utilización. El rendimiento del extracto acuoso liofilizado expresado a partir del alga fresca para Bryothamnion triquetrum y Halimeda opuntia fue de 3,95 \% y 3,40 \% respectivamente

\section{Determinación del contenido de polifenoles}

La determinación del contenido de polifenoles totales se realizó por el método de Folin-Ciocalteau según Vidal et al., 12. El contenido de polifenoles totales se expresó como $\mu \mathrm{g}$ equivalentes de ácido gálico (EAG)/g de extracto acuoso liofilizado y se estimó a partir de una curva de calibración obtenida en el intervalo de 4,7-600 $\mu$ g de ácido gálico.

\section{Determinación del poder reductor}

El poder reductor para ambos extractos acuosos fue evaluado siguiendo el protocolo descrito por Oyaizu ${ }^{13}$. Se mezcló $1 \mathrm{~mL}$ de muestra con 2,5 mL de tampón fosfato $(0,2 \mathrm{M}, \mathrm{pH}$ $6,6)$ y $2,5 \mathrm{~mL}$ de $\mathrm{K}_{3} \mathrm{Fe}(\mathrm{CN})_{6}$ al $1 \%$. Se incubó a $50^{\circ} \mathrm{C}$ durante $20 \mathrm{~min}$. Se dejó enfriar y se añadieron $2,5 \mathrm{~mL}$ de ácido tricloroacético al $10 \%$. Se centrifugó a 800 g (Heal Force Beckman GS-GKR, USA) durante 10 minutos y se tomaron alícuotas de 2,5 $\mathrm{mL}$ de la capa superior y se les añadieron 0,5 $\mathrm{mL}$ de $\mathrm{FeCl}_{3}$ al 0,1 \% y 2,5 mL de agua destilada. Finalmente se midió la DO a 720 nm (Rayleigh VIS-723G, China). Los incrementos en la DO se consideraron como incrementos en el poder reductor.

El intervalo de concentraciones evaluado para los extractos de las dos algas fue de $2-128 \mathrm{mg} / \mathrm{mL}$. Como compuesto antioxidante de referencia se empleó el ácido ascórbico a una concentración de $1 \mathrm{mg} / \mathrm{mL}$.

Determinación de la capacidad atrapadora de radicales libres mediante el ensayo de decoloración del radical DPPH*

La evaluación de la capacidad atrapadora de radicales $\mathrm{DPPH} \bullet$ de los extractos de Bryothamnion triquetrum y Hali- 
meda opuntia se realizó siguiendo el protocolo descrito por Brand-Williams et al., ${ }^{14}$, con algunas modificaciones. En una placa de 96 pocillos se añadieron $20 \mu \mathrm{L}$ de los correspondientes extractos en un intervalo de concentraciones de 0,31 a $60 \mathrm{mg} / \mathrm{mL}$, y adicionalmente $200 \mu \mathrm{L}$ de solución de $\mathrm{DPPH}^{\bullet}$ a $125 \mu \mathrm{M}$ en una mezcla de metanol: propanol (1:1, v/v). Posteriormente se midió la DO a $517 \mathrm{~nm}$ en un lector de placas (PolarStar Omega, Alemania) con agua destilada como referencia. El antioxidante de referencia utilizado fue vitamina $C$ en un intervalo de concentraciones de 0,06 a1 $\mathrm{mg} / \mathrm{mL}$.

La actividad secuestradora del extracto de B. triquetrum y H. opuntia fue expresada empleando el parámetro porcentaje de inhibición de la absorbancia del $\mathrm{DPPH}^{\bullet}\left(\mathrm{PI}_{\mathrm{DPPH}} \cdot\right)$, según la ecuación:

$\mathrm{PI}_{\mathrm{DPPH}} \cdot(\%)=\left[1-\left(\mathrm{DO}_{\text {muestra }} / \mathrm{DO}_{\mathrm{ref}}\right)\right] * 100$

Donde:

$\mathrm{DO}_{\text {muestra }}$ : DO $(517 \mathrm{~nm})$ de la muestra a los 30 minutos de reacción

$\mathrm{DO}_{\text {ref }}:$ DO (517 nm) del control de DPPH•

\section{Capacidad inhibitoria de la peroxidación lipídica es- pontánea en homogenado de cerebro de rata}

La evaluación de la capacidad inhibitoria de la peroxidación lipídica espontánea en homogenado de cerebro de rata fue llevada a cabo según el protocolo descrito por Ohkawa et al., ${ }^{15}$.

La preparación del homogenado de cerebro de rata se realizó de la siguiente manera: ratas Wistar machos fueron decapitadas y los cerebros extraídos se pesaron, inmediatamente lavados con $\mathrm{NaCl}$ 0,9 \% frío y homogenizados en un homogenizador de cuchillas en una relación 1:5 (p: v) en tampón fosfato (50 mM, pH 7,4). Los homogenados fueron centrifugados a $800 \mathrm{~g}$ a $4^{\circ} \mathrm{C}$ durante $15 \mathrm{~min}$ (Heal Force Beckman GS-GKR, USA). Los sobrenadantes obtenidos se conservaron a $-70^{\circ} \mathrm{C}$ hasta su utilización.

Se incubaron $25 \mu \mathrm{L}$ de homogenado de cerebro con igual volumen de muestra, a $37^{\circ} \mathrm{C}$ durante 40 minutos en baño con oscilación constante. Posteriormente se adicionaron $350 \mu \mathrm{L}$ de ácido acético al $20 \%$, pH 3,5 y $600 \mu \mathrm{L}$ de ácido tiobarbitúrico al 0,5\%. Los viales se incubaron durante una hora a $85^{\circ} \mathrm{C}$ y transcurrido ese tiempo se adicionaron $50 \mu \mathrm{L}$ de SDS al $10 \%$. Se centrifugó a 800 g durante 15 min a temperatura ambiente (Heal Force Beckman GS-GKR, USA) y se midió la DO a 532 nm (Rayleigh VIS-723G, China).

La determinación de la máxima lipoperoxidación espontánea se midió empleando tampón fosfato $50 \mathrm{mM}$, pH 7,4 en lugar de la muestra. El control de lipoperoxidación inicial, el cual no se incubó en baño, se le adicionó $25 \mu \mathrm{L}$ de tampón fosfato $50 \mathrm{mM}, \mathrm{pH}$ 7,4, en sustitución de la muestra. La DO obtenida para este control fue sustraída al resto de las determinaciones. Las concentraciones de los extractos evaluadas estuvieron entre 0.16 a 10 . Como control positivo se empleó ácido ascórbico a $1 \mathrm{mg} / \mathrm{mL}$.

La actividad antioxidante fue expresada como porcentaje de inhibición de la peroxidación lipídica (PI peroxidación lipídica) según la ecuación:

$\mathrm{PI}$ peroxidación lipídica $(\%)=\left[1-\left(\mathrm{DO}_{\text {muestra }} / \mathrm{DO}_{\text {ref }}\right)\right]^{* 100}$

Donde:

$\mathrm{DO}_{\text {muestra }}$ : DO (532 nm) de la muestra

$\mathrm{A}_{\text {ref }}: \mathrm{DO}(532 \mathrm{~nm})$ de la referencia (máxima lipoperoxidación espontánea)

\section{Capacidad inhibitoria de la hemólisis inducida por AAPH}

En este ensayo se empleó sangre de carnero, colectada en tubos con EDTA como anticoagulante. La sangre se centrifugó a 4000 rpm durante 10 minutos (Heal Force Beckman GS-GKR, USA) y se lavó el precipitado con 5 volúmenes de PBS a pH 7,4. El lavado de los eritrocitos se repitió 3 veces y el precipitado fue suspendido en 4 volúmenes de PBS.

La determinación de la actividad anti-hemolítica de los extractos se realizó según la metodología descrita por Aman et al., ${ }^{16}$ con algunas modificaciones. Se mezclaron $0,5 \mathrm{~mL}$ de suspensión eritrocitaria, $0,5 \mathrm{~mL}$ de extracto a diferentes concentraciones y 0,5 mL de AAPH [dihidrocloruro de 2,2-azobis (2-metil-amidinopropano)] $200 \mathrm{mM}$ y se mantuvo con agitación constante a $37^{\circ} \mathrm{C}$ durante $3 \mathrm{~h}$. Después de la incubación, la mezcla se diluyó en 8 volúmenes de PBS y se centrifugó a $4000 \mathrm{rpm}$ durante $5 \mathrm{~min}$. Finalmente se tomó el sobrenadante y se midió la absorbancia a $540 \mathrm{~nm}$ (Rayleigh VIS-723G, China).

El intervalo de concentraciones evaluado para los extractos acuosos de las algas fue de $2-128 \mathrm{mg} / \mathrm{mL}$. Como antioxidante de referencia se empleó el ácido ascórbico en una concentración de $0,25-1 \mathrm{mg} / \mathrm{mL}$ disuelto en agua destilada. Se realizaron los siguientes controles:

- Control de estabilidad de los eritrocitos: 0,5 mL de suspensión eritrocitaria con $1 \mathrm{~mL}$ de PBS.

- Control de estabilidad de los eritrocitos frente a la muestra: $0.5 \mathrm{~mL}$ de suspensión eritrocitaria, $0,5 \mathrm{~mL}$ de la muestra (con la máxima concentración utilizada) y 0,5 $\mathrm{mL}$ de PBS. 
- Control de hemólisis con AAPH: 0,5 mL de suspensión eritrocitaria, $0,5 \mathrm{~mL}$ de AAPH y $0,5 \mathrm{~mL}$ de PBS.

- Control de hemólisis total: 0,5 mL de suspensión eritrocitaria y $1 \mathrm{~mL}$ de agua destilada.

Como antioxidante de referencia se empleó el ácido ascórbico a una concentración de $1 \mathrm{mg} / \mathrm{mL}$ disuelto en PBS.

La actividad antioxidante fue expresada como porcentaje de inhibición de la hemólisis inducida por el AAPH $\left(\mathrm{PI}{ }_{\text {hemólisis }}\right)$ según la ecuación:

$\mathrm{PI}_{\text {hemólisis }}(\%)=\left[\left(\mathrm{A}_{\mathrm{AAPH}}-\mathrm{A}_{\text {muestra }}\right) / \mathrm{A}_{\mathrm{AAPH}}\right]$ * 100

Donde:

$\mathrm{A}_{\mathrm{AAPH}}:$ absorbancia con AAPH a $540 \mathrm{~nm}$

$\mathrm{A}_{\text {muestra: }}$ : absorbancia de la muestra a evaluar

\section{Ensayo de toxicidad aguda con Artemia salina}

El protocolo para este ensayo se realizo de acuerdo al procedimiento descrito por Meyer et al., ${ }^{17}$. Los quistes fueron puestos a eclosionar en un recipiente con agua de mar, a temperatura ambiente durante 24 horas bajo régimen continuo de luz. Los nauplios se colectaron y trasladaron a los pozos de la placa de ensayo utilizando una pipeta Pasteur. El número de larvas colectadas y añadidas a la placa se determinó utilizando un microscopio estereoscópico (NOVEL).

Grupos de 10 nauplios por pocillo se expusieron a concentraciones de 10, 50 y $100 \mathrm{mg} / \mathrm{mL}$ de los extractos de $B$. triquetrum y $H$. opuntia durante 24 horas a temperatura ambiente y bajo régimen continuo de luz. Cada ensayo se realizo por triplicado. Al finalizar las 24 horas de exposición, se contó el número de organismos muertos y se calculó el porcentaje de mortalidad. Las larvas se consideraron muertas si no exhibían movimiento durante varios segundos de observación al microscopio estereoscópico. El experimento se consideró válido si el porcentaje de mortalidad en los controles (pozos preparados e incubados en las mismas condiciones pero en ausencia de extractos) no excedió el $10 \%$.

El grado de toxicidad de los extractos se definió en función del intervalo en que se encontraron los valores de $\mathrm{CL}_{50}$ de acuerdo con las categorías siguientes: extremadamente tóxico $\left(\mathrm{CL}_{50}<10 \mu \mathrm{g} / \mathrm{mL}\right)$, muy tóxico $\left(10<\mathrm{CL}_{50}<100\right)$, moderadamente tóxico $\left(100<\mathrm{CL}_{50}<1000\right)$ y no tóxico $\left(\mathrm{CL}_{50}>\right.$ $1000 \mu \mathrm{g} / \mathrm{mL}$ ).

\section{Análisis Estadístico}

Todos los experimentos se realizaron por triplicado y los resultados fueron expresados como media \pm desviación estándar $(\mathrm{X} \pm \mathrm{DE})$ de tres experimentos independientes. Para el procesamiento de los datos y graficado de los resultados se empleó el asistente matemático Origin 7.0 y para el análisis estadístico se utilizó el programa Statistica 8.0. Previo al procesamiento estadístico de los resultados, se comprobó en todos los experimentos, la normalidad de los datos y la homogeneidad de varianza. Se realizaron comparaciones entre las medias de dos grupos por la Prueba t de Student. Las diferencias significativas entre grupos se determinaron por análisis de varianza simple (ANOVA) y en caso de existir diferencias se aplico el test paramétrico de Tukey. Valores $\mathrm{p}<0,05$ fueron considerados estadísticamente significativos.

\section{RESULTADOS}

\section{Contenido de polifenoles}

El contenido total de compuestos polifenólicos para los extractos acuosos de Bryothamnion triquetrum y Halimeda opuntia fue 51,21 $\pm 2,25$ y 19,99 $\pm 1,12 \mu \mathrm{g}$ EAG/ mg de extracto liofilizado respectivamente.

\section{Capacidad reductora de los extractos de Bryothamnion triquetrumy Halimeda opuntia}

Como se muestra en la Figura 1, en el intervalo de concentraciones evaluadas para los extractos de ambas algas se observa una actividad reductora dosis-dependiente. Estos resultados sugieren para ambos extractos la capacidad de reducir el estado de transición del hierro y consecuentemente la generación de radicales libres. El extracto acuoso de $B$. triquetrum presentó mayor capacidad reductora, a partir de $5 \mathrm{mg} / \mathrm{mL}$ se observaron valores de poder reductor estadísticamente superiores $(p<0,005)$ con respecto a $H$. opuntia. Se empleó como control positivo de actividad antioxidante al ácido ascórbico con una absorbancia de 2,824 $\mathrm{nm}$ a una concentración de $1 \mathrm{mg} / \mathrm{mL}$.

Figura 1. Capacidad reductora expresada en función de mg de extracto acuoso liofilizado de Bryothamnion

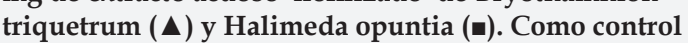
positivo se utilizó ácido ascórbico a $1 \mathrm{mg} / \mathrm{mL}(\circ)$. El ensayo fue realizado de acuerdo a Oyaizu et al., (1986). Los resultados están expresados como $\pm \pm D E, n=3$.

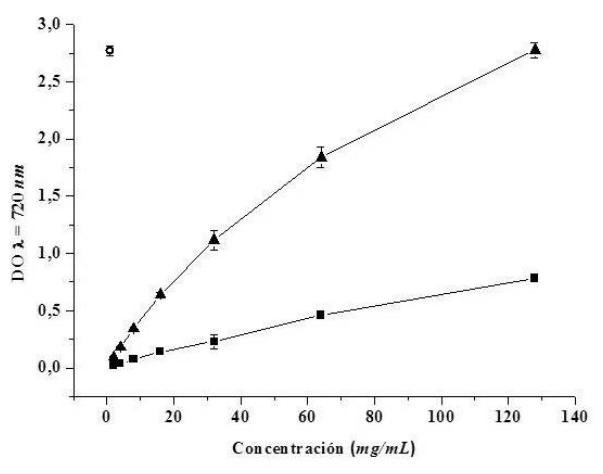


Actividad antioxidante con el ensayo de atrapamiento de radicales $\mathrm{DPPH}^{*}$

Los resultados de la actividad atrapadora de radicales $\mathrm{DPPH}$ de los extractos de las algas se muestran en la Figura 2. Ambos extractos presentan elevada capacidad de atrapar radicales DPPH, con una relación dosis-actividad dependiente. El extracto de B. triquetrum produjo la decoloración total $(100 \%)$ de los radicales DPPH a la concentración de 5 $\mathrm{mg} / \mathrm{mL}$ mientras que el extracto de $H$. opuntia requirió cuatro veces esa concentración. Los valores de $\mathrm{CI}_{50}$ obtenidos para los extractos de $B$. triquetrum y $H$. opuntia fueron de $1,35 \pm 0,09 \mathrm{mg} / \mathrm{mL}$ y $12,05 \pm 0,67 \mathrm{mg} / \mathrm{mL}$ respectivamente, siendo el primero aproximadamente diez veces más potente respecto al segundo.

Figura 2. Actividad atrapadora de radicales DPPH • expresada en función de mg de extracto acuoso liofilizado de Bryothamnion triquetrum ( $\Delta$ ) y Halimeda opuntia ( $\square$ ). El ensayo fue realizado de acuerdo a Goupy et al., (1999). Los resultados están expresados como $x \pm D E, n=3$.

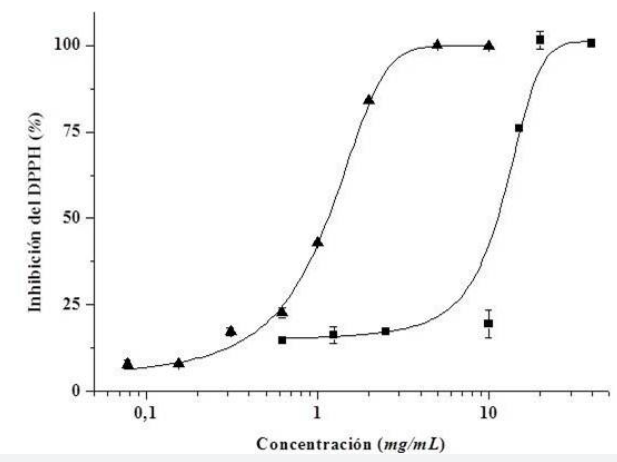

Figura 3. Inhibición de la lipoperoxidación espontánea en homogenados de rata expresado en función de $\mathrm{mg}$ de extracto acuoso liofilizado de Bryothamnion triquetrum ( $\Delta$ ) y Halimeda opuntia ( $($ ). Como control positivo se utilizó ácido ascórbico a $1 \mathrm{mg} / \mathrm{mL}(\circ)$. El ensayo fue realizado de acuerdo a Ohkawa et al., (1999). Los resultados están expresados como $x \pm D E, n=3$.

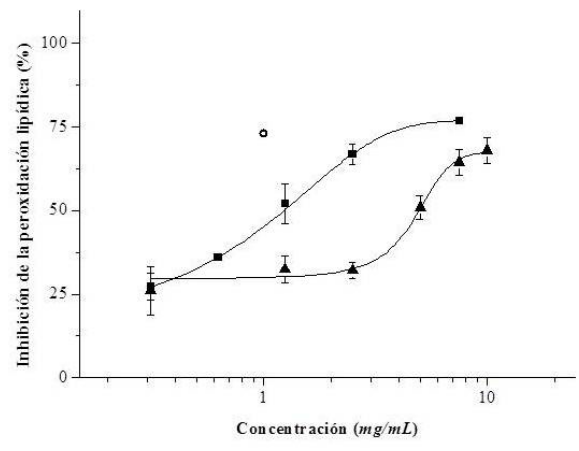

Capacidad inhibitoria de la peroxidación lipídica espontánea en homogenado de cerebro de rata

El efecto de los extractos acuosos de Bryothamnion triquetrum y Halimeda opuntia sobre la peroxidación lipídica espontánea en homogenado de cerebro de rata se presenta en la Figura 3. Ambos extractos son capaces de inhibir la peroxidación lipídica espontánea con una relación directamente proporcional a la dosis. A partir de los resultados obtenidos en este ensayo se calcularon las $\mathrm{CI}_{50}$ de la peroxidación lipídica espontánea para ambos extractos con valores de 4,96 $\pm 0,37$ y 1,23 $\pm 0,26 \mathrm{mg} / \mathrm{mL}$ para B.triquetrum y $H$. opuntia respectivamente. El cálculo de este parámetro reveló que el extracto acuoso de $H$. opuntia fue aproximadamente 5 veces mas potente que el extracto acuoso de $B$. triquetrum.

\section{Actividad inhibitoria de la hemólisis inducida por} AAPH

Los extractos acuosos de ambas algas resultaron efectivos al inhibir la hemólisis de los eritrocitos provocada por el radical libre AAPH (Figura 4). La actividad antihemolítica fue dosis-dependiente, hasta el valor de concentración 25 $\mathrm{mg} / \mathrm{mL}$ para el alga $H$. opuntia y $50 \mathrm{mg} / \mathrm{mL}$ para B. triquetrum. A partir de $25 \mathrm{mg} / \mathrm{mL}$ se observan valores de actividad antihemolítica estadísticamente superiores para el extracto del alga H opuntia.

Capacidad inhibitoria de la hemólisis inducida por AAPH expresado en función de $\mathrm{mg}$ de extracto acuoso liofilizado de Bryothamnion triquetrum ( $\square$ ) y Halimeda opuntia ( $\square$ ). A: Dosis $2-30 \mathrm{mg} / \mathrm{mL}$ B: Dosis $35-128 \mathrm{mg} / \mathrm{mL}$

El ensayo fue realizado de acuerdo a Aman et al., (2013). Como antioxidante de referencia se empleó el ácido ascórbi-

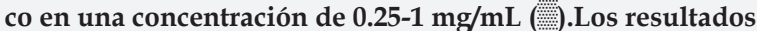
están expresados como $x \pm D E, n=3$.

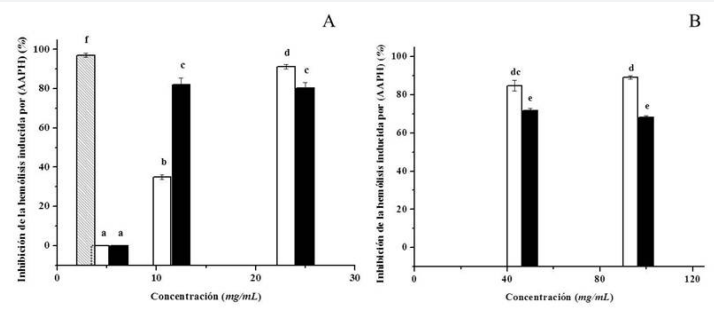

Ensayo de toxicidad aguda con Artemia salina

En la Tabla I se muestran los resultados obtenidos para el estudio de toxicidad con Artemia salina para los extractos acuosos de Bryothamnion triquetrum y Halimeda opuntia. En este experimento solo se observa mortalidad con el extracto de H. opuntia en la última concentración estudiada. 
Tabla I. Estudio de toxicidad aguda por el método de la Artemia salina de los extractos acuosos liofilizados de Bryothamnion triquetrum y Halimeda opuntia. El ensayo fue realizado de acuerdo (Meyer, 1982). Cada extracto liofilizado se disolvió en agua de mar hasta obtener la concentración deseada. El experimento se realizó por triplicado $y$ en cada pocillo $n=10$ nauplios

\begin{tabular}{|c|c|c|}
\hline \multirow{2}{*}{$\begin{array}{l}\text { Concentración } \\
\text { (mg/mL) }\end{array}$} & \multicolumn{2}{|l|}{ Mortalidad (\%) } \\
\cline { 2 - 3 } & $\begin{array}{l}\text { Bryothamnion } \\
\text { triquetrum }\end{array}$ & Halimeda opuntia \\
\hline 10 & 0 & 0 \\
\hline 50 & 0 & 0 \\
\hline 100 & 0 & 30 \\
\hline
\end{tabular}

\section{DISCUSIÓN}

En la actualidad se ha incrementado el interés por los extractos vegetales como fuentes de compuestos antioxidantes, al prevenir el daño celular por inactivación de los radicales libres y en este contexto las algas constituyen excelentes candidatos para la obtención de compuestos bioactivos ${ }^{6}$.

Las propiedades antioxidantes de los extractos acuosos de las algas roja Bryothamnion triquetrum y verde Halimeda opuntia se evaluaron a través de algunos de los ensayos más utilizados, con el objetivo de comparar sus propiedades antioxidantes y su relación con el contenido en polifenoles y adicionalmente esclarecer sus posibles mecanismos de acción.

\section{Contenido de polifenoles}

Los polifenoles constituyen uno de los más numerosos y representativos grupos de metabolitos secundarios de las plantas, con propiedades beneficiosas para la salud humana-. Estos metabolitos tienen la capacidad de neutralizar radicales libres, debido a la habilidad de donar los átomos de hidrógeno de los grupos hidroxilos presente en su estructura de anillos aromáticos, la capacidad de atrapar radicales libres, funciones como agentes quelantes y la inducción de enzimas antioxidantes ${ }^{18}$.

Diferentes investigadores han demostrado la presencia de compuestos fenólicos en las algas marinas y su relación con las propiedades antioxidantes ${ }^{6}$. De acuerdo a Kuda e Ikemori ${ }^{19}$ el contenido de polifenoles de las algas marinas varía considerablemente aun en especies dentro del mismo género, desde muy bajos contenidos hasta valores altos.

El contenido de polifenoles del extracto acuoso de Bryothamnion triquetrum fue superior a los valores obtenidos por Vidal et al., ${ }^{12}$ (8,05 mg de EAG/ g de extracto seco), aunque estos investigadores emplearon un extracto acuoso con diferente esquema de extracción. Este valor de polifenoles totales para $B$ triquetrum fue aproximadamente 2,6 veces superior al obtenido para el alga verde $H$. opuntia. Zubia et al.,$^{20}$ encontraron una relación similar (3,3 veces) del contenido de polifenoles de las algas B. triquetrum y $H$. monile.

El contenido de polifenoles de H. opuntia en este trabajo fue superior a los obtenidos por Silva et al., ${ }^{11}$ con un extracto acuoso de esta especie de alga (97.2 $7.3 \mu \mathrm{g}$ EAG/ g) y Batista-González et al., ${ }^{10}$ con un extracto acuoso del alga $H$. monile $(179,45 \pm 18,54 \mu \mathrm{g} / \mathrm{g}$ de extracto seco). Batista-González et al. ${ }^{10}$ reportaron un valor similar de polifenoles totales (74.3 mg of polifenoles/g alga seca) en un estudio sobre la actividad antioxidante de Halimeda spp con fracciones de ácidos fenolicos. Costa-Mugica et al., ${ }^{21}$ encontraron valores de polifenoles totales para el alga Halimeda incrassata de 131 $\mu \mathrm{g}$ GAE/g alga seca.

En estudios previos se identificaron como los principales componentes de la fracción polifenolica del alga B.triquetrum a los ácidos $p$-cumárico, $t$-cinámico y ferúlico y se relacionó la actividad antioxidante de $B$. triquetrum con estos compuestos ${ }^{7,12,}$ mientras que para $H$. opuntia se identificaron y cuantificaron 8 ácidos fenólicos y cinámicos, resultando el componente mayoritario el ácido salicílico?. Otros investigadores encontraron cantidades apreciables de compuestos polifenolicos en especies del genero Halimeda. Yoshie et al., ${ }^{22}$ han identificado ácido caféico en $H$. macroloba mientras Vidal et al., ${ }^{23}$ encontraron cantidades apreciables de acido salícilico y en menor proporción otros ácidos fenólicos y cinámicos en H incrassata.

Nakai et al., ${ }^{24}$ evaluaron la actividad antioxidante de diferentes especies de algas y en $H$. opuntia encontraron actividad atrapadora de radicales hidroxilos $(\mathrm{OH})$, explicando las propiedades antioxidantes por la presencia de florotaninos y terpenoides. Otros metabolitos con función antioxidante, que en alguna medida pudieran influir en esta propiedad son los carotenos y el ácido ascórbico, también presentes en B. triquetrum ${ }^{22}$. Zubia et al., ${ }^{25}$ señalan que los bromofenoles presentes en las algas rojas $B$. byssoides y $E$ lanosa con similar ubicación taxonómica que $B$. triquetrum (Familia Rhodomelaceae, orden CERAMIALES), pudieran ser los responsables de su alta actividad antioxidante. Vidal et al., ${ }^{12}$ detectaron cantidades apreciables de compuestos bromados en el alga B. triquetrum.

\section{Evaluación de la capacidad reductora de los extractos de Halimeda opuntia y Bryothamnion triquetrum}

Diferentes investigadores señalan que los compuestos reductores tienen la capacidad de romper las reacciones en 
cadenas de los radicales libres por la donación de un átomo de hidrógeno y de esta manera exhiben propiedades antioxidantes ${ }^{26}$.

En el intervalo de concentraciones evaluadas para los extractos de ambas algas se observa una alta capacidad de reducir el estado de transición del $\mathrm{Fe}^{3+}$ y consecuentemente la generación de radicales libres de manera dosis-dependiente.

Los valores de capacidad reductora obtenidos con el extracto de Halimeda opuntia resultaron similares a los valores encontrados en la literatura para el género Halimeda. Silva et al., ${ }^{11}$, obtuvieron un valor de absorbancia igual a $0,1 \mathrm{~nm}$ con una concentración de $10 \mathrm{mg} / \mathrm{mL}(\square=700 \mathrm{~nm}$ ) con un extracto acuoso de $H$. opuntia, valor similar al obtenido en este trabajo a esa concentración. Batista-González et al., ${ }^{10}$ trabajando con fracciones polares de ácidos fenolicos de $H$ monile obtuvieron una DO de $0.13 \mathrm{~nm}$ con $20 \mu \mathrm{g}$ de polifenoles totales, valores que convertidos en $\mathrm{mg} / \mathrm{mL}$ también son comparables a los resultados de este trabajo.

Al comparar los valores de absorbancia a una concentración de $128 \mathrm{mg} / \mathrm{mL}$, en el extracto de $B$. triquetrum se obtuvo una DO de $2.798 \mathrm{~nm}$ mientras que con el extracto de H.opuntia el valor fue de $0.800 \mathrm{~nm}$, de manera que B.triquetrum resulto 3,5 veces más eficiente en la reducción de los iones $\mathrm{Fe}^{3+}$ a $\mathrm{Fe}^{2+}$ que H. opuntia. Este resultado puede ser explicado por un mayor contenido de polifenoles en este extracto (aproximadamente 2,5 veces mayor con respecto a $H$. opuntia) y la estrecha relación que existe entre este parámetro y la capacidad reductora de extractos naturales. Kuda e Ikemori ${ }^{19}$ observaron una buena correlación entre contenido de polifenoles y la capacidad reductora en 12 especies de algas.

Se empleó como control de actividad antioxidante el ácido ascórbico en una concentración de $1 \mathrm{mg} / \mathrm{mL}$, con una DO de 2,824 nm, valor de absorbancia igual al observado con el extracto de $B$. triquetrum y aproximadamente 3,5 veces superior al extracto de $H$. opuntia pero a una concentración 128 veces inferior. Sin embargo si consideramos que comparamos un compuesto químicamente puro (ácido ascórbico) con un extracto crudo podremos concluir que los extractos presentan una actividad antioxidante relativamente alta.

\section{Ensayo de actividad atrapadora de radicales DPPH}

Dentro de los mecanismos de acción antioxidante mas importantes de las algas marinas se encuentra la capacidad atrapadora radicales libres y por este motivo, el ensayo de inactivación del radical DPPH es una de las metodologías más utilizadas para estudiar sus propiedades antioxidantes $^{26}$. El DPPH es un radical estable coloreado que puede aceptar protones donados por entidades antioxidantes presentes en estos extractos convirtiéndose en su forma no radicalaria incolora.

Los extractos acuosos de ambas algas provocaron la decoloración total, llegando a la máxima actividad de atrapamiento de radicales DPPH. El extracto de B. triquetrum logró inhibir el $100 \%$ del radical a la concentración de $5 \mathrm{mg} / \mathrm{mL}$ mientras que a esa concentración el extracto de $H$. opuntia produjo un $24 \%$ de inhibición. Al comparar estadísticamente los valores de $\mathrm{CI}_{50}$ obtenidos con esta metodología (1,15 \pm 0,06 y $11,34 \pm 0.30 \mathrm{mg} / \mathrm{mL}$ para $B$. triquetrum y H. opuntia respectivamente), observamos que el alga $B$. triquetrum resulta aproximadamente cuatro veces más potente que $H$. opuntia, resultado que concuerda con lo obtenido en el experimento de la capacidad reductora. También Boonchum et al., ${ }^{27}$ encontraron un valor de $\mathrm{CI}_{50}$ de $0.837 \mathrm{mg} / \mathrm{mL}$ para un extracto acuoso de Halimeda macroloba

El valor obtenido con el extracto acuoso del alga $B$. triquetrum es superior al informado por Vidal et al., ${ }^{23}$ que obtuvieron con $4 \mathrm{mg} / \mathrm{mL}$ un $38 \%$ de inhibición del DPPH y también superior a lo reportado por Zubia et al., ${ }^{20}$ con una $\mathrm{CI}_{50}$ de $12.9 \mathrm{mg} / \mathrm{mL}$ para esta especie de alga. Los resultados de $H$. opuntia se corresponden con valores informados por otros investigadores. Silva et al., ${ }^{11}$ reportaron un $48 \%$ de inhibición con $7 \mathrm{mg} / \mathrm{mL}$ de extracto acuoso liofilizado de H. opuntia.

En esta investigación encontramos valores relativamente altos de polifenoles para el alga $B$. triquetrum, por lo que es de esperar que presente una capacidad atrapadora de radicales libres mayor que $H$. opuntia. Adicionalmente estos resultados evidencian que existe una relación directa entre el contenido de polifenoles de los extractos acuosos y las propiedades antioxidantes en función de la capacidad reductora y el atrapamiento de radicales libres, siendo $B$. triquetrum más potente en estos sistemas que H. opuntia.

Zubia et al., ${ }^{25}$ estudiando las propiedades antioxidantes con el ensayo de DPPH y poder reductor de 24 algas rodófitas observaron una correlación significativa entre la actividad atrapadora del radical DPPH y el poder reductor en las algas rojas evidenciado en que ambos ensayos se explican porque se basan en la donación de electrones/hidrógenos.

Las propiedades antioxidantes de un extracto vegetal están en dependencia al radical libre al que se enfrenta la molécula antioxidante. Kaur et al., ${ }^{28}$ demostraron que la actividad atrapadora de radiales libres de un extracto de flores de Cassia siamea difieren en dependencia del radical al que se enfrenta el extracto, para el radical DPPH se obtuvo un 100 $\%$ de inhibición a una concentración que no fue tan eficaz para otros radicales como $\mathrm{O}_{2}{ }^{--} \mathrm{y} \mathrm{OH}^{\bullet}$, lo que pudiera ser ex- 
plicado porque el mecanismo de atrapar DPPH está estrechamente relacionado al poder reductor, por la capacidad de donar un protón, mientras para los otros radicales se fundamenta en el atrapamiento. Este criterio podría corroborar los resultados de este trabajo, ya que la peroxidación lipídica espontánea está mediada principalmente por las EROs y pudiera resultar que $B$. triquetrum, más potente en cuanto a mecanismo de donar protones, no sea tan eficiente para atrapar radicales libres a diferencia de $H$. opuntia. Vidal et al., ${ }^{23}$ demostraron que la actividad antioxidante de B. triquetrum puede ser explicada en parte por la capacidad atrapadora de radical $\mathrm{OH}^{\bullet}$ y la dismutación de radicales $\mathrm{O}_{2} \cdot$ -

\section{Inhibición de la lipoperoxidación espontánea en homo- genado de cerebro de rata}

La peroxidación lipídica es un evento de daño celular, donde se afectan los lípidos poli-insaturados de las membranas, formándose productos citotóxicos como el trans4-hidroxi-2-nonenal (HNE) y el malondialdehído (MDA), compuestos que a su vez desencadenan un estrés oxidativo a nivel de las biomembranas lo que puede conducir a la despolarización y permeabilización no selectiva de las mismas y cambios estructurales así como alteraciones a las proteínas embebidas en ella, de ahí que este constituya un evento de efectos altamente deletéreos para la célula ${ }^{26}$.

Los resultados de este trabajo evidencian que ambos extractos de algas inhiben efectivamente la generación de TBARS con $\mathrm{CI}_{50}$ de 5,09 $\pm 0,25$ y 1,25 $\pm 0,31 \mathrm{mg} / \mathrm{mL}$ para B. triquetrum y H. opuntia respectivamente. Al comparar estadísticamente los valores de $\mathrm{CI}_{50}$ se hace evidente que el alga $H$. opuntia es mucho más efectiva que $B$. triquetrum. Batista-González et al., ${ }^{10}$ estudiando un extracto acuoso de H opuntia encontraron un valor de $\mathrm{CI}_{50}$ similar a lo obtenido en este trabajo. Rivero et al., ${ }^{8}$ investigando las propiedades antioxidantes de un extracto acuoso de Halimeda incrassata reportaron valores de máxima efectividad en la inhibición de la peroxidación lipídica espontánea en homogenados de cerebro de rata a la concentración de $5 \mathrm{mg} / \mathrm{mL}$. En este trabajo para el alga Halimeda opuntia se obtuvo a esa misma concentración aproximadamente un 75\% de inhibición de la peroxidación lipídica. Nuestros resultados también concuerdan con Vidal et al., ${ }^{12}$ quienes investigando extractos de $B$. triquetrum observaron una $\mathrm{CI}_{50}$ de inhibición de la lipoperoxidacion en cerebro de ratas de $6,46 \mathrm{mg} / \mathrm{mL}$

Algunas moléculas pueden inhibir la lipoperoxidacion por mecanismos como la prevención de la iniciación de la oxidación de cadenas de ácidos grasos, enlazamiento de iones metálicos de transición catalíticos, y descomposición de peróxidos y además en adición el atrapamiento de radicales libres. En los ensayos de capacidad reductora y atrapamiento de radical libre DPPH, el extracto acuoso del alga $B$. triquetrum había mostrado resultados superiores con respecto al extracto de $H$. opuntia, sin embargo en este ensayo se obtuvo mayor inhibición de la lipoperxidacion espontánea con el extracto acuoso de H. opuntia. Lim et al., ${ }^{29}$ reportaron mayor valor de $\mathrm{CI}_{50}$ para el ensayo de $\mathrm{DPPH}$ con respecto a valor obtenido en el ensayo de inhibición de la lipoperoxidación en homogenados de cerebro con un extracto de N. aculeate, lo que concuerdan con nuestros resultados. Matsukawa et al., ${ }^{30}$ no encontraron correlación entre la inhibición de lipo-oxigenasa y la actividad atrapadora de DPPH, sugiriendo que estas actividades ocurren con mecanismos no relacionados. En el ensayo de atrapamiento del radical DPPH, los extractos de algas actúan como donadores de electrones y/o hidrogeno mientras que en la inhibición de la lipo-oxigenasa se pudiera bloquear la adición enzimática de oxígeno al ácido graso (sustrato de la enzima) y por tanto inhibir la formación de hidroperóxido. En estudios previos de estos autores, demostraron una correlación entre el consumo de oxígeno y la formación de hidroperóxidos durante el proceso de inhibición de la lipo-oxigenasa. Adicionalmente la diferencia en respuesta de acuerdo al método empleado también se pudiera explicar por las diferencias cuantitativitas en las composiciones químicas y/o en tipos de compuestos polifenólicos de estas algas por lo que resulta lógico que presenten diferentes propiedades antioxidantes como por ejemplo algunos mecanismos entre los que se comprenden la quelación de hierro (en la etapa de iniciación) o un incremento sinérgico de la actividad antioxidante de vitamina E en la etapa de propagación. Este criterio está en concordancia con resultados obtenidos por Yoshie et al., ${ }^{22}$ quienes demostraron que existían diferencias en cuanto a la composición de polifenoles y las cantidades de estos en extractos naturales de algas incluso dentro del mismo género. Otra explicación pudiera ser, la presencia de otros componentes en el extracto pudieran actuar de forma sinérgica con los estos y potenciar la actividad inhibitoria en la peroxidación lipídica espontánea ${ }^{7}$.

En este trabajo se encontró una relación directa entre la inhibición de la lipoperoxidación y el contenido de polifenoles para las dos algas. Otros autores ${ }^{8,10}$ también han encontrado una relación directa entre esta actividad antioxidante y el contenido de polifenoles. Chakraborty et al., ${ }^{31}$ sugieren que la inhibición de la peroxidación lipidica de extractos de Turbinaria spp puede ser debida a la presencia de compuestos polifenólicos que disrumpen la reacción en cadena de los radicales libres por donación de un protón al radical acido graso y de esa manera inhibir la peroxidación lipídica..

Lim et al., ${ }^{32}$ estudiando fracciones del alga Sargassum siliquastrum no encontraron una relación directa entre el conte- 
nido de polifenoles y la actividad antioxidante sin embargo encontraron una relación estrecha entre la inhibición de la peroxidación lipídica y la hemólisis de eritrocitos inducida por AAPH, concluyendo que una de las fracciones estudiada probablemente contenía un potente antioxidante bloqueador de la rupturas de cadenas no polares.

\section{Capacidad inhibitoria de la hemólisis inducida por el AAPH}

AAPH es un radical peroxilo que inicia el proceso peroxidativo, generando a la vez otros radicales libres para inducir oxidación de los ácidos grasos y proteínas, ocasionando un daño sobre la organización de los eritrocitos y conduciendo eventualmente a lisis de la membrana ${ }^{33}$. Adicionalmente la liberación del hierro desde los hematíes puede aumentar el efecto pro-oxidante de hidroperóxidos provenientes de la reacción del oxígeno y el AAPH, con un papel importante como agente catalítico redox de acuerdo a la reacción de Fenton y de Haber-Weiss.

Los resultados de este trabajo (Figura 4A) evidencian que ambos extractos protegen a los eritrocitos del efecto toxico del radical AAPH sobre las biomembranas de una manera dosis-dependiente. El extracto de H. opuntia resulta más potente ya que a bajas concentraciones $(12.5 \mathrm{mg} / \mathrm{mL})$ mostró un $82.2 \%$ de inhibición frente a un $35.1 \%$ con el extracto de B. triquetrum. Estos resultados se encuentran en concordancia con los resultados del ensayo de inhibición de la peroxidación lipídica, lo que resulta lógico si consideramos que ambas metodologías estudian fundamentalmente las propiedades antioxidantes de una molécula para prevenir los efectos tóxicos producidos por un radical libre sobre los lípidos de las biomembranas. Estos resultados concuerdan con los obtenidos por Lim et al., ${ }^{32}$, quienes trabajando con extractos del alga Sargassum siliquastrum encontraron una excelente correlación entre la inhibición de la peroxidación lipídica y la protección contra hemólisis de eritrocitos, considerando como responsables de estas actividades a los compuestos fenólicos.

En la literatura existen pocos reportes de estudios de propiedades antioxidantes de extractos vegetales mediante este ensayo. Benites et al., ${ }^{34}$ demostraron que concentraciones superiores a $0,2 \%(\mathrm{p} / \mathrm{v})$ de extractos de frutas inhibían la hemólisis en aproximadamente un $40 \%$. Sin embargo para el extracto de $H$. opuntia a concentraciones superiores a $25 \mathrm{mg} / \mathrm{mL}$ (Figura 4B) se comienza a observar una disminución de la protección frente a la actividad hemolítica del $\mathrm{AAPH}$, a diferencia de lo que ocurre con el aumento de las concentraciones de $B$. triquetrum, donde el incremento de las concentraciones no disminuye sus propiedades antioxidantes protectoras y/o incremento de efectos tóxicos. Simi- lares resultados encontró Lim et al., ${ }^{32}$ (2002) trabajando con diferentes extractos del alga Sargassum siliquastrum, quienes encontraron una satisfactoria actividad antihemolitica de los eritrocitos de rata en las concentraciones de 0,2 a 10 $\mu \mathrm{g} / \mathrm{mL}$, sin embargo con la concentración de $50 \mu \mathrm{g} / \mathrm{mL}$ la protección disminuía aproximadamente 9 veces. En determinadas condiciones experimentales los compuestos polifenólicos pueden actuar como pro-oxidantes en presencia de $\mathrm{Fe}^{3+}$, dada su habilidad para enlazarse y reducir el $\mathrm{Fe}^{3+}$ vía transferencia electrónica colateral, aspecto corroborado por Puppo ${ }^{35}$ quien observo un incremento en la producción de $\bullet \mathrm{OH}$ estudiando el efecto de los flavonoides sobre la formación de radicales hidroxílicos por la reacción de Fenton. De manera que un factor a considerar seria la cantidad de $\mathrm{Fe}$, consecuentemente un incremento de la cantidad del extracto conlleva un incremento de polifenoles y a su vez del Fe presente en este extracto. Adicionalmente es conocido que el genero Halimeda contiene cantidades apreciables de este mineral. Anantharaman et al., ${ }^{36}$ investigaron la composición de minerales y de metales trazas de 9 especies de algas (incluidas H.macroloba y H.tuna), resultando el Fe como el segundo mineral en contenido para todas las especies de algas, con las mayores concentraciones en Halimeda macroloba. Otra explicación acerca de la toxicidad observada en las dosis altas de $H$. opuntia puede ser el aumento en las concentraciones de determinados metabolitos secundarios presentes en esta especie de alga, lo que generaría una actividad pro-oxidante tanto de los polifenoles, vitamina $\mathrm{C}$ y la posible presencia de metales de transición $\left(\mathrm{Fe}^{2+}, \mathrm{Cu}^{+}\right.$, $\mathrm{Zn}^{2+}$ ), que en presencia de radicales peroxilo inducido por el AAPH aumentarían el daño oxidativo por generación de radicales $\mathrm{OH}$.

En este sistema experimental (protección al eritrocito) actúan a la vez dos de los mecanismos de acción que explican la actividad antioxidante de extractos de algas: la capacidad de atrapar radicales libres y de inhibir la peroxidación de lipídica. Hipotéticamente se podría postular otro mecanismo de acción, la capacidad quelante de $\mathrm{Fe}$, entonces los resultados para cada extracto con este ensayo estarán en función de la composición química de moléculas que puedan contribuir más o menos a cada uno de estos mecanismos. Al parecer, la mayor actividad antihemolítica de $H$. opuntia con respecto a $B$. triquetrum se pudiera deber a mecanismos de atrapamientos de radicales libres más eficientes y a la inhibición de la peroxidación lipídica, independientemente del contenido de polifenoles que presenta y además la quelación de $\mathrm{Fe}^{3+}$.

\section{Ensayo de toxicidad aguda con Artemia salina}

Dentro de los ensayos actuales de evaluaciones toxicológicas con métodos alternativos se encuentra la toxicidad agu- 
da con Artemia salina, muy utilizado para evaluar extractos vegetales. Con este método, diferentes autores ${ }^{17}$ consideran valores de la $\mathrm{CL}_{50}$ superiores a $1000 \mu \mathrm{g} / \mathrm{mL}$ como extractos no tóxicos. Al comparar la toxicidad de estas algas se puede observar que $H$. opuntia resulta más toxica que $B$. triquetrum, en el intervalo de concentraciones evaluadas para las dos algas (10-100 mg/mL). Solo se observó mortalidad con el extracto de H. opuntia en la dosis máxima estudiada (100 $\mathrm{mg} / \mathrm{mL}$ ) lo que permite clasificar a estos extractos como no tóxicos. Los resultados de este trabajo coinciden con lo reportado por Freile-Pelegrin et al., ${ }^{37}$ quienes estudiaron diferentes especies de algas marinas mexicanas, entre ellas Bryothamnion triquetrum, Halimeda tuna y Halimeda incrassata y encontraron valores de $\mathrm{CL}_{50}$ por encima de $1000 \mu \mathrm{g} /$ mL. Ara et al., ${ }^{38}$ evaluaron un extracto etanólico de Halimeda tuna, y observaron una mortalidad de un $30 \%$ con $5 \mathrm{~g} /$ $\mathrm{mL}$, explicada por la presencia de metabolitos secundarios utilizados por el alga como mecanismo de defensa frente a depredadores.

En los estudios de toxicidad resulta importante considerar el tipo de extracción; generalmente los extractos acuosos de las algas marinas resultan menos tóxicos aunque exhiban propiedades antioxidantes satisfactorias. Freile-Pelegrin et al., ${ }^{37}$ estudiaron la toxicidad mediante el ensayo de A. salina de 17 especies de algas con diferentes tipos de extracciones y de todos, los menos tóxicos eran los extractos acuosos.

Es importante resaltar que los extractos de $B$. triquetrum y $H$ opuntia exhiben propiedades antioxidantes a concentraciones por debajo de 20-30 mg/mL en los diferentes ensayos estudiados, de manera que posibles efectos tóxicos pudieran ocurrir a concentraciones muy superiores a aquellas donde actúa como antioxidantes.

\section{CONCLUSIONES}

Las algas marinas constituyen organismos con perspectivas alentadoras como fuentes de bioactivos, con disímiles aplicaciones tanto en la prevención como en el tratamiento de diversas enfermedades. Diferentes autores han comprobado que los extractos de algas marinas presentan propiedades antioxidantes explicadas por una amplia variedad de moléculas, lo que a su vez determina que estos extractos posean diferentes mecanismos de acción. En este trabajo se comprobó que las algas Bryothamnion triquetrum y Halimeda opuntia poseen propiedades antioxidantes debidas al menos en parte a su contenido de polifenoles, con mayor cantidad de compuestos polifenólicos en el alga B. triquetrum.

$\mathrm{Al}$ analizar detenidamente los resultados de los ensayos de actividad antioxidante, se observa que el alga $B$ triquetum posee mayores potenciales como atrapadora de radicales libres aunque pueden estar presentes otras moléculas con otros mecanismos de acción, sin embargo el alga $H$ opuntia ofrece mayores perspectivas como inhibidor de los procesos lipoperoxidativos y en general en la protección de biomembranas. Adicionalmente resulta importante señalar que las dos algas poseen una baja toxicidad.

En resumen, se puede concluir que las algas $B$ triquetrum y $H$ opuntia resultan promisorias fuentes de antioxidantes naturales con aplicaciones como fitofármaco y/o nutracéutico.

\section{REFERENCIAS}

1. Mac Artain P, Gill CIR, Brooks M, Campbell R, Rowland IR. Nutritional value of edible seaweeds. Nutr Rev. 2007; 65(12): 535-43.

2. Roig JT. Plantas medicinales, aromáticas o venenosas de Cuba. $1^{\text {a }}$ ed. Cuba: Editorial Ciencia y técnica, La Habana; 1974.

3. Proksch P, Edrada-Ebel RA, Ebel R. Drugs from the sea- Opportunities and obstacles. Mar Drugs. 2003; 1: 5-17.

4. Gomez -Gutierrez CM, Guerra-Rivas G, Soria-Mercado IE, Ayala-Sánchez NE. Marine edible algae as disease preventers. Adv Food Nutr Res. 2011; 64: 29-39.

5. Sampth-Wiley P, Neefus CD, Jahnke LS. Seasonal effects of sun exposure and emersion on intertidal seaweed physiology: fluctuations in antioxidant contents, photosynthetic pigments and photosynthetic efficiency in the red alga PhorphyraumbilicalisKutzing (Rodophyta, Bangiales). J Exp Mar Biol Ecol. 2008; 36: 83-91.

6. Dutra Rocha F, Crespo Pereira R, Coelho Kaplan MA, Laneuville Teixeira V. Produtos naturais de algas marinhas e sue potencial antioxidante. Braz J Pharmacogn. 2007;17(4): 631-39.

7. Fallarero A, Peltoketo A, Loikkanen JJ, Tammela P, Vidal A, Vuorela P. Effects of aqueous extracts of Bryothamnion triquetrum on chemical hypoxia and aglycemia-induced damage in GT1-7 mouse hypothalamic immortalized cells. Phytomedicine 2006; 13(4):240-45.

8. Rivero F, Fallararo A, Castañeda O, Dajas F, Manta E, Areces A, et al. Antioxidant activity in vivo and in vitro of Halimeda incrassata aqueous extracts. Cienc Tecnol Alimentos (Campinas) 2003; 23: $256-63$.

9. Vidal A, Silva de Andrade-Wartha ER, de Oliveira e Silva AM, Pavan R, Lima A, Fallarero A, et al. Actividad antioxidante y polifenoles de algas marinas verdes Halimedaopuntiay Halimeda monile. Ars Pharm. 2009; 50 (1): 24-31.

10. Batista-Gonzalez AE, de Oliveira e Silva AM, Vidal-Novoa A, Pinto JR, Portari Mancini DA, Mancini-Filho J. Analysis of antioxidant properties of hydrophilic fractions from seaweed Halimeda monileL. and its function in vivo. J Food Biochem. 2012; 36 :189-97.

11. Silva AM de O, Vidal-Novoa A, Batista-González AE, Pinto JR, Portari Mancini DA, Reina-Urquijo W, et al. Antioxidant 
activity and hepatoprotectivie properties of polyphenols in vitro and in vivo from seaweeds Halimeda opuntia (Linnaeus) Lamouroux. Redox report. 2012;17 (2): 47-53.

12. Vidal A, Motidome M, Mancini J, Fallarero A, Tanae MM, Brandao LM, et al. Actividad antioxidante y ácidos fenólicos del alga marina Bryothamnion triquetrum (SG Gmelim) Howe. Braz J Pharm Sci. 2001; 37(3), 373-82.

13. Oyaizu M. Studies on products of browning reaction: Antioxidant activities of products of browning reaction prepared from glucosamine. Jpn J Nutr. 1986; 44: 307-15.

14. Brand-Williams W, Cuvelier ME, Berset C. Use of a Free Radical Method to Evaluate Antioxidant Activity. Lebensm.-Wiss. u.-Technol. 1995; 28:25-30.

15. Ohkawa H, Noburo O, Kunio Y. Assay for lipid peroxides in animal tissues by thiobarbituric acid reaction. Anal Biochem. 1979; 95: 351-58.

16. Aman S, Moin S, Owanis M, Siddiqui MU. Antioxidant activity of thymol: protective role in AAPH-induced hemolysis in diabetic erythrocytes. IJPSI, 2013; 2 (3): 55-60.

17. Meyer BN, Ferrigni NR, Putnam JE, Jacobsen LB, Nichols DE, McLaughlin JL. Brine shrimp: a convenient general bioassay for active plant constituents. Planta Med. 1982; 45:31-4.

18. Vauzour D, Rodriguez-Mateos A, Corona G, Oruna-Concha MJ, Spencer JPE. Polyphenols and human health: prevention of disease and mechanisms of action. Nutrients. 2010; 2: 110631.

19. Kuda T, Ikemori T. Minerals, polysaccharides and antioxidant properties of aqueous solutions obtained from macroalgal beach-casts in the Noto Peninsula, Ishikawa, Japan. Food Chem. 2009; 112: 575-81.

20. Zubia M, Robledo D, Freile-Pelegrin Y. Antioxidant activities in tropical marine macroalgae from the Yucatan Peninsula, Mexico. J Appl Phycol. 2007; 18: 449-58.

21. Costa-Mugica A, Batista-Gonzalez AE, Mondejar D, Soto-López Y, Brito-Navarro V, Vázquez AM, et al. Inhibition of LDLoxidation and antioxidant properties related to polyphenol content of hydrophilic fractions from seaweed Halimeda Incrassata (Ellis) Lamouroux. Braz J Pharm Sci. 2012; 48 (1): 31-7.

22. Yoshie $Y$, Wang W, Hsieh YP, Suzuki T. Compositional difference of phenolic compounds between two seaweeds, Halimeda spp. J Tokyo Univ Fish.. 2002; 88: 21-4.

23. Vidal A, Fallarero A, de Andrade-Wartha ERS, de Oliveira e Silva AM, de Lima A, Pavan R, et al. Composición química y actividad antioxidante del alga marina roja Bryothamnion triquetrum (S.G.Gmelin) Howe. Braz J Pharm Sci. 2006; 43(4): 589-600.

24. Nakai M, Kageyama N, Nakahara K, Miki W. Phlorotannins as radical scavengers from the extract of Sargassum ringgoldianum. Mar Biotechnol. 2006; 8: 409-14.
25. Zubia M, Fabre MS, Kerjean V, Deslandes E. Antioxidant and cytotoxic activities of some red algae (Rhodophyta) from Brittany coasts (France) Bot Mar. 2009; 52: 268-77.

26. Moon J-K, Shibamoto T. Antioxidant assays for plant and food components. J Agric Food Chem. 2009; 57(2): 1655-66.

27. Boonchum W, Peerapornpisal Y, Kanjanapoth D, Pekkoh J, Pumas C, Jamjai U, et al. Antioxidant activity fo some seaweed from the Gulf of Tailand. Int J Agric Biol. 2011; 13(1):95-9.

28. Kaur G, AlamS, Jabbar Z, Javed K, Athar M. Evaluation of antioxidant activity of Cassia siamea flowers. J Ethnopharmacol. 2006; 108:340-8

29. Lim CS, Jin DQ, Sung JY, Lee JH, Choi HG, Há I, et al. Antioxidant and anti-inflamatory activities of the methanolic extract of Neorhodomela aculeate in Hippocampal and Microglial cells. Biol Pharm Bull. 2006; 29(6): 1212-6.

30. Matsukawa R, Dubinsky Z, Kishimoto E, Masaki K, Masuda $\mathrm{Y}$, Takeuchi $\mathrm{T}$, et al. A comparison of screening methods for antioxidant activity in seaweeds. J Appl Phycol. 1997; 9: 29-35.

31. Chakraborty K, Praveen NK, Vijayan KK, Rao GS. Evaluation of phenolic contents and antioxidant activities of brown seaweeds belonging to Turbinaria spp. (Phaeophyta, Sargassaceae) collected from Gulf of Mannar. Asian Pac J Trop Biomed. 2013; 3(1):8-16.

32. Lim SN, Cheung PCK, Ooi VEC, Ang PO. Evaluation of antioxidative activity of extracts from a brown seaweed, Sargassum siliquastrum. J. Agric Food Chem. 2002; 50:3862-6.

33. Pannangpetch $\mathrm{P}$, Laupattarakasem P, Kukongviriyapan V, Kukongviriyapan U, Kongyingyoes B, Aromdee C. Antioxidant activity and protective effect against oxidative hemolysis of Clinacanthus nutans (Burm.f) Lindau. Songklanakarin. J Sci Technol. 2007; 29 (Suppl. 1): 1-9.

34. Benites Vílchez J, Díaz García R, López Vivar J, Gajardo Solari S, Kusch Fuschlocher F, Rojas Arredondo M. Actividad antioxidante y antibacteriana de seis cáscaras de frutos del oasis de Pica. Biofarbo. 2011; 19(1):1-7.

35. Puppo A. Effect of flavonoids on hydroxyl radical formation by Fenton-type reactions; influence of the iron chelator. Phytochemistry. 1992; 31: 85-8.

36. Anantharaman P, Karthikaidevi G, Manivannan G, Thirumaran G, Balasubramanian T. Mineral composition of marine macroalgal from Mandapam coastal regions; Southeast Coast of India. Rec Res Sci Tech. 2010; 2(10): 66-71.

37. Freile-Pelegrin $Y$, Robledo D, Chan-Bacab MJ, Ortega-Morales $\mathrm{BO}$. Antileishmanial properties of tropical marine algae extracts. Fitoterapia. 2008; 79: 374-7.

38. Ara J, Sultana V, Ehteshamul-Haque S, Qasim R, Viqar Uddin Ahmad VU. Cytotoxic activity of marine macro-algae on Artemia salina (Brine Shrimp). Phytother Res. 1999; 13: 304-7. 